\title{
Perspectives
}

\section{Antigen Receptor Genes As Molecular Markers of Lymphoid Neoplasms}

\author{
Stanley J. Korsmeyer \\ Division of Hematology-Oncology, Departments of Medicine, Microbiology, and Immunology, Howard Hughes Medical Institute, \\ Washington University School of Medicine, St. Louis, Missouri 63110
}

\section{Introduction}

Molecular analysis of human lymphoid neoplasms is markedly altering our concepts of their pathogenesis and classification. Before the era of recombinant DNA, major advances were provided by the investigation of cell surface antigens representing maturational stages of B or T cells (1). Despite the generation of many antibodies to $B$ and $T$ cell antigens, it was still impossible to classify the cellular type of some lymphoid neoplasms. This inadequacy frequently reflected the admixture of large numbers of nonneoplastic cells within lymphomatous tissue. Such cells could not be distinguished inasmuch as virtually all the lineageassociated markers were present on normal as well as malignant cells. In other instances, leukemias represented stages of development prior to the expression of lineage-restricted antigens. Frequently, it was even impossible to determine whether a lymphoid neoplasm was of clonal origin.

The rearrangements of DNA which assemble the antigenspecific receptor genes in B or T cells provide the necessary tools to overcome many of these limitations. The immunoglobulin (Ig) and T cell receptor (TCR) ${ }^{1}$ genes are composed of multiple, separated gene subsegments within their germline or embryonic state. During lymphoid development a DNA recombination process assembles the components of Ig genes in B cells or TCR genes in T cells (2-4). Most of our knowledge concerning these genes including their ordered sequence of DNA rearrangements, mechanisms of recombination, somatic mutation, alternative RNA splicing, and transcriptional regulation was gleaned from established lymphoid neoplasms. In return, these contributions to basic biology have paid dividends by resolving uncertainties concerning the lineage commitment, clonality, stage of development, and pathogenesis of leukemias and lymphomas. Specifically, Ig and TCR gene rearrangements create DNA-level markers unique to each individual cell. This sensitive and specific molecular fingerprint is capable of identifying a clonal expansion of B or T cells. Moreover, a developmental hierarchy for both Ig and TCR gene rearrangements occurs during early $B$ and $T$ cell maturation, providing new means of categorizing neoplasms. Most importantly, unanticipated rearrangements of Ig and TCR loci have been discovered which directly contribute to the ma-

Received for publication 3 September 1986.

1. Abbreviations used in this paper: ALL, acute lymphoblastic leukemia; T-ALL, acute lymphoblastic leukemia of the T cell type; TCR, T cell receptor.

J. Clin. Invest.

(c) The American Society for Clinical Investigation, Inc.

0021-9738/87/05/1291/05 \$1.00

Volume 79, May 1987, 1291-1295 lignant phenotype. This class of recombinations occurs between nonhomologous chromosomes and translocates a cellular oncogene into the Ig or TCR locus.

\section{Somatic rearrangement of gene subsegments generates antigen receptor diversity}

An individual can generate a seemingly unlimited number $\left(10^{6}\right.$ to $10^{9}$ ) of different antibody and TCR specificities. The central process in receptor diversification is a somatic rearrangement of the DNA subsegments comprising the variable portion of these molecules. Both members of the super-Ig gene family share the same structural motif in which genetic information for the antigen-binding portion of the molecule exists as discontinuous gene subsegments entitled variable $(\mathrm{V})$, joining $(\mathrm{J})$, and at times diversity (D) segments (Fig. 1). Each of these gene subsegments is flanked by an evolutionarily conserved set of signals which help mediate their rearrangement. This recombination occurs early in B or T cell development to juxtapose coding segments creating contiguous $\mathrm{V} / \mathrm{J}$ or $\mathrm{V} / \mathrm{D} / \mathrm{J}$ rearrangements. Each Ig locus (heavy chain, $\kappa$ and $\lambda$ light chain) and each TCR locus $(\alpha, \beta$, and $\gamma$ ) possess a multitude of $\mathrm{V}$ regions, an alternative set of $\mathrm{J}$ segments, and in some cases families of $\mathrm{D}$ regions. The choice of segments is relatively stochastic so that each individual B or $T$ cell generates a unique pattern of receptor rearrangements. These varied rearrangements can be readily identified as altered sized DNA restriction fragments on a Southern blot (Fig. 1).

\section{Ig and TCR rearrangements as uniformly applicable clonal} markers

The determination that a malignancy is of clonal origin has often been used as a diagnostic aid to distinguish benign from malignant proliferations (5). Historically, assessing the clonality of lymphoid neoplasms was predominantly limited to mature B cell tumors that displayed the exclusive presence of but one lightchain isotype ( $\kappa$ or $\lambda$ ) (6). No comparable set of alternatively expressed chains was identified on $T$ cells. A population of normal $B$ or $T$ cells is polyclonal in nature and possesses numerous different Ig or TCR gene rearrangements. None of these are above the threshold of detectability by Southern blot analysis (7). In contrast, a monoclonal proliferation represents the progeny of a single cell, and all cells of that clone possess the same, identifiable DNA rearrangement pattern specific to the tumor (8).

The detection of an Ig or TCR rearrangement is a relatively sensitive as well as specific marker, capable of identifying even minority populations (1-5\%) of clonal cells in tissues of mixed cellularity. For example, analysis of TCR and Ig genes has uncovered minority populations of neoplastic B cells in malignant lymph nodes in which $T$ cells predominated, thus enabling the correct diagnosis to be made (8). This approach has also dis- 


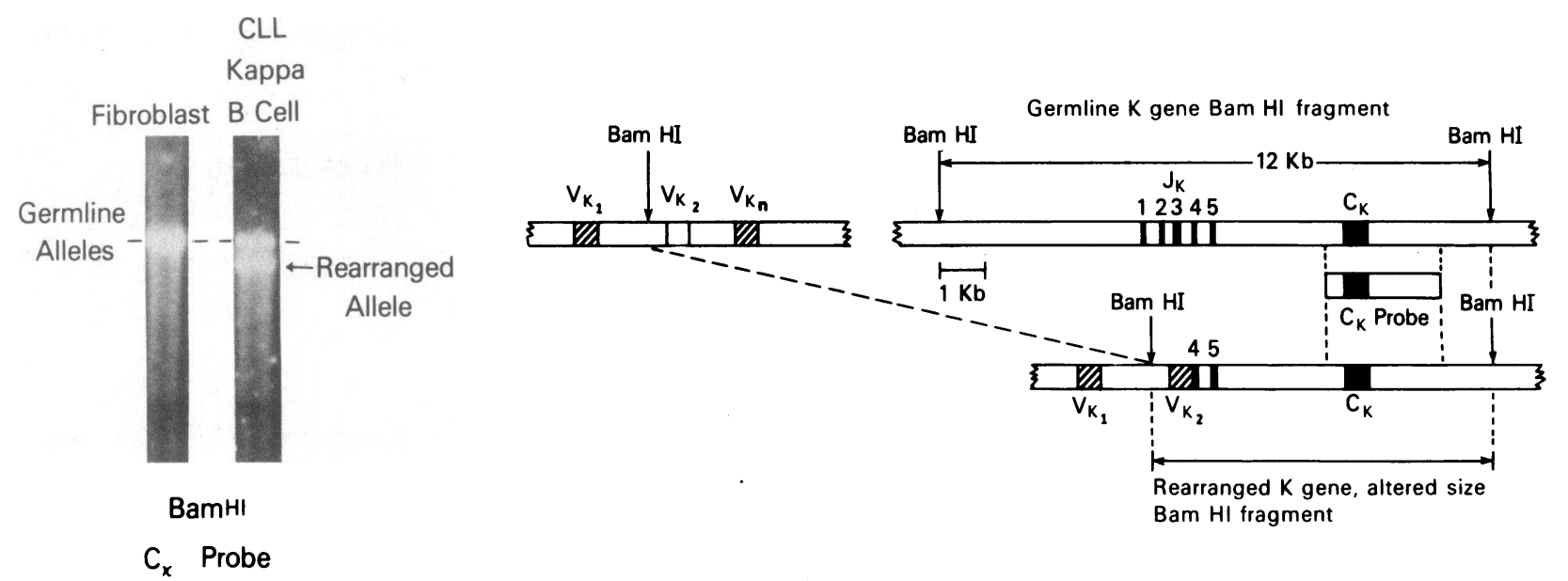

Figure 1. A schematic presentation of the human $\kappa$ gene locus is shown at the right with multiple variable $\left(V_{k}\right)$ regions, five functional joining $\left(J_{k}\right)$ regions, and but a single constant $\left(C_{k}\right)$ segment. A DNA rearrangement juxtaposes a $V_{k}$ and $J_{k}$ region on an activated allele re-

covered clonal B cell subpopulations in lymph nodes in which the histologic impression was a benign hyperplasia or in lymphoproliferations felt to be polyclonal $(8,9)$. Consequently, it should be stressed that the determination of clonality is not necessarily tantamount to a verdict of malignancy. This is particularly true in the setting of immunodeficiency in which lymphoproliferation can result in transient clones, but these clones may still be under some regulatory control. Importantly, the accompanying clonal rearrangements are unique to these cells and provide the capacity to follow the natural history of such lymphoproliferations. As these DNA level markers do not require the expression of a final product, they are applicable to the entire spectrum of neoplasia including pre-B and pre-T cells.

\section{Developmental hierarchy of Ig and TCR rearrangements}

Mature B cells that bear surface Ig possess the obligate Ig heavyand light-chain rearrangements $(8,10)$. Similarly, mature $T$ cells bearing the cell surface $\mathrm{T} 3$ molecule have in general rearranged and expressed the $\alpha$ and $\beta$ TCR genes to form the $\alpha, \beta$ heterodimer that completes the T3-Ti (idiotype) complex (11-16). Pre$B$ and pre-T cell clonal populations representing early differentiation at the time of gene joining were discovered in acute lymphoblastic leukemia (ALL). Before the use of gene rearrangements the majority of ALLs were referred to as "non-T, non-B" because they failed to express $\mathrm{T}$ cell surface antigens and also lacked surface Ig. However, Ig genes analysis revealed that these leukemias represented distinct stages of $B$ cell precursor development. The earliest pre-B cells had rearranged heavy chains, but retained germline light-chain genes, establishing a heavy before light-chain order. Moreover, the light-chain gene rearrangement pattern indicated a $\kappa$ before $\lambda$ usage $(7,17,18)$. If initial attempts to make a $\kappa$ gene were unsuccessful, the $\kappa$ gene complex was eliminated by a novel recombinatorial segment termed the " $\alpha$-deleting element" $(\kappa \mathrm{de})$, before the $\lambda$ light-chain genes were used. Indeed, human pre-B cell leukemias provided a clonal expansion of cells at the appropriate stage of development that allowed the molecular cloning of this $k$-deleting element (19).

An examination of fetal thymus and ALL of the T cell type (T-ALL) also revealed a developmental sequence to TCR rear- sulting in the introduction of a new $5^{\prime}$ Bam $\mathrm{HI}$ site. The $C_{\kappa}$ probe sees an altered size Bam HI fragment on the rearranged vs. the germline allele as demonstrated by the Southern blot at the left.

rangements (20-24). The $T$ cell precursor forms of ALL indicated that the $\beta$ TCR genes were activated early in intrathymic ontogeny before the $\alpha$ TCR genes (23-24). Most T-ALLs were of adequate maturity to have rearranged their $\beta$-chain gene and express it. However, only one half of the ALLs were of sufficient maturity to express the $\alpha$ TCR. This more mature subset is capable of expressing a complete T3-Ti complex. Most T-ALLs with $\beta$ TCR rearrangements have also rearranged $\gamma$ TCR genes although few still express $\gamma$. Exceptional cells were noted that rearranged $\gamma$ but not $\beta$, or in contrast, rearranged $\beta$ but not $\gamma$ (24). Thus the earliest events in TCR activation appear not to be invariant. Furthermore, rare T-ALLs exist that possess the T3 molecule but fail to express the classic $\alpha$ plus $\beta$ TCR heterodimer (24). These T-ALLs provide an important opportunity to characterize $T$ cell subsets that utilize novel TCR complexes $(25,26)$.

\section{Gold standards of lineage determination}

The validity of utilizing Ig and TCR gene rearrangements to assign $B$ or $T$ cell lineage depends in part upon the lineage restriction of these events. Unfortunately, lineage spillover of certain types of gene rearrangement clearly occurs. Intermediate $(D / J)$ rearrangements of Ig heavy-chain genes have been noted in $\sim 10 \%$ of $T$ cell neoplasms in addition to some nontransformed $T$ cell lines $(18,27,28)$. Conversely, rearrangement of $\alpha$ and $\beta$ TCR genes and even expression of $\alpha$ have been noted in $B$ cell lineage neoplasms $(12,24,29)$. The $\alpha$ and $\beta$ events in $B$ cells are at a comparably low frequency. However, pre-B cell ALLs displayed a surprisingly high incidence $(45 \%)$ and a rather unique pattern of $\gamma$ TCR rearrangement (24). The higher incidence of this TCR rearrangement in pre-B cell ALLs may reflect the maturational arrest of these cells at an early stage of maturation when rearrangements of both types of receptor genes might be allowed. The canonical recombinational signals (heptamer [CACAGTG]-spacer-nonamer [ACAAAAACC]) that flank these gene subsegments are highly homologous in Ig and TCR genes and appear to be the recognition template for a common recombinase capable of rearranging either Ig or TCR genes (30). The crossover of both Ig and TCR gene rearrangements into the opposite lineage is apparently a normal event and represents 
remnants of an early decision-making process that occurs prior to absolute lineage commitment (31).

The lack of complete lineage fidelity of Ig and TCR gene rearrangements requires some caution when using these markers to assign a lineage designation. Ig light-chain gene rearrangement as a later event in maturation has to date been restricted to the $B$ cell series. However, the most immature pre-B cells will have only Ig heavy chain rearrangement. In this situation the presence of the B cell-associated surface antigens B4, B1, and HLA-DR provides useful confirmatory data $(18,32)$. The absence of TCR rearrangement is compatible with B cell commitment, but some cells that are otherwise pre-B cells will have rearranged especially the $\gamma$ TCR gene. Conversely, the activation of TCR loci is a prerequisite for a $\mathrm{T}$ cell assignment. The lack of Ig heavy- and light-chain rearrangement is an important aid to $T$ cell lineage determination. When Ig heavy-chain and TCR genes are simultaneously rearranged the assessment of surface antigens is required. The combination of Ig gene, TCR gene, and surface antigen determinations can correctly assign lineage in nearly all leukemias and lymphomas. The rare exceptions to this doctrine include genetically uncommitted progenitors lacking either Ig or TCR rearrangement and provide the substrate to assess molecular events preceding gene recombination (24).

\section{Tumor differentiation and clonal evolution}

The pre-B and T cell ALLs are maturationally arrested clones that revealed some of the earliest knowledge of human lymphocyte differentiation. Individual cases of pre-B and pre-T cell leukemia are not always static either, but can display developmental progressions. For example, two separate lymphoid blast crises in a chronic myelogenous leukemia patient both arose from a common lymphoid progenitor because they shared identical Ig heavy rearrangements. However, one episode had progressed to $\lambda$ light-chain rearrangement while the other retained germline light-chain genes. Thus, despite being neoplastic these cells were capable of a normal differentiative event (33). Similarly, Abelson murine leukemia virus-transformed pre-B cells have been shown to undergo genetic progression from heavy to light chain and for DJ intermediate to completed V/D/J rearrangements (34).

ALLs and lymphomas have also been noted to display variations in Ig gene patterns over time. The emergence of these different rearrangements has raised the possibility that these tumors might be biclonal (35). Do these changes reflect the emergence of truly separate tumors with unique transformation events in a host predisposed to such malignancies? Alternatively, do they result from evolving subclones of a single malignant progenitor? By analyzing multiple clonal markers including translocation breakpoints as well as all possible receptor rearrangements, most of these changes can be shown to represent evolving tumor heterogeneity (36-38). Variations in Ig genes are often secondary rearrangements occurring at a mature B cell stage. Perhaps mature B cell neoplasms can actually reactivate recombinase to produce these additional rearrangements. Idiotypic variation has also been noted to result from well-documented somatic mutation mechanisms (39). All of these examples of tumor variation may be attempts to escape from immunoregulatory control by altering one target of regulation, the antigen receptor.

\section{Minimal residual disease}

A major remaining challenge in leukemia research is to detect minimal residual disease in the hope of identifying the subset of patients who will subsequently relapse. Ig and TCR rearrangements have the requisite specificity to cleanly identify persistent or recurrent leukemic clones in the midst of large numbers of normal cells. The major question is whether the sensitivity of this technique $(1-5 \%)$ is sufficient to detect disease before histopathologic evidence of relapse. Pre-B ALL patients examined during early induction phases of chemotherapy revealed that those lacking lymphoblasts had returned to germline Ig gene patterns (36). This indicates that therapy actually eliminates leukemic cells rather than simply altering their morphology or maturation. The most crucial group to follow are those patients receiving maintenance chemotherapy who are felt to be in clinical remission because some of these cases will relapse. Clonal Ig gene rearrangements have been noted in occasional patients during such presumed remissions and those followed longitudinally relapsed $(36,40)$. Important clinical information is also provided when gene rearrangements are absent in bone marrows suspicious for relapse (unexplained lymphocytosis, focal aggregation of lymphoblasts, or slightly elevated [2-6\%] lymphoblasts) (36). Other rearrangements that are unique to tumor cells including defined breakpoints of chromosomal translocations will make this approach to minimal residual disease feasible in nonlymphoid cancer. Technical innovations that amplify the sensitivity beyond the $1-5 \%$ range yet retain specificity will be important to make this an effective clinical tool.

\section{Antigen receptor genes are the sites of chromosomal translocation}

The association of specific chromosomal translocations with histologically distinct neoplasms prompted the thesis that genes flanking these breakpoints would participate in malignant transformation (41). Within lymphoid neoplasms the same genes that normally rearrange to create antigen receptors (Ig in B cells, TCR in T cells) also mediate interchromosomal translocations. Unexpected rearrangements of these loci have provided a bridge to walk across such chromosomal translocations to the opposite side and identify new genes. Burkitt lymphoma was the first instance in which the chromosomal breakpoint was placed at the Ig gene loci (heavy chain at $14 \mathrm{q} 32, \kappa$ at $2 \mathrm{p} 11$ or $\lambda$ at $22 \mathrm{q} 11$ ) (42-46). The other partner was uniformly the c-myc cellular oncogene at $8 \mathrm{q} 24$. The introduction of c-myc next to an Ig gene locus altered the regulatory control of this gene contributing to uncontrolled growth (47).

Of interest, chromosomal breakpoints at $14 \mathrm{q} 32$ occur repeatedly in other mature B cell neoplasms (48). A translocation between $11 \mathrm{q} 13$ and $14 \mathrm{q} 32$ has been noted in multiple myeloma and other B cell neoplasms. The most common lymphoma translocation typifies follicular lymphoma and places a new B cell-associated gene from chromosome segment 18q21 into 14q32 (Fig. 2). Several groups have documented that the breakpoints focus at the $5^{\prime}$ ends of $\mathrm{J}_{\mathrm{H}}$ regions on the $14 \mathrm{q}^{+}$chromosome (49-51), whereas the breakpoints on the $18 \mathrm{q}^{-}$chromosome are at the $3^{\prime}$ ends of $D_{H}$ regions (52). The recombinatorial signals are eliminated and " $N$ " segment extranucleotides are introduced at the site of chromosomal juncture just as occurs in normal $\mathrm{D} / \mathrm{J}$ rearrangements (49-52). Despite the fact that follicular lymphomas are phenotypically mature B cells, these findings indicate that the translocation occurs at the $D / J$ rearrangement step early in pre-B cell development. The site of chromosomal breakage is also focused on chromosome 18 . Over $70 \%$ of $t(14 ; 18)$ translocations occur within a 2.8-kb major breakpoint region and most of these cluster within 150 base pairs $(37,52)$. No 


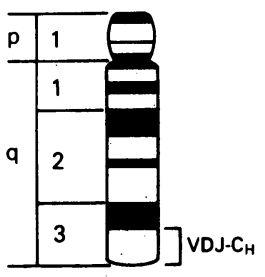

14

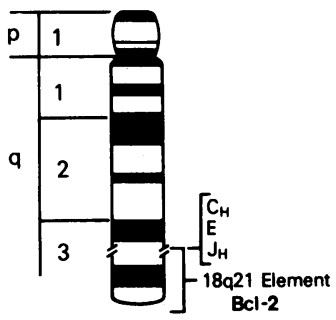

der 14

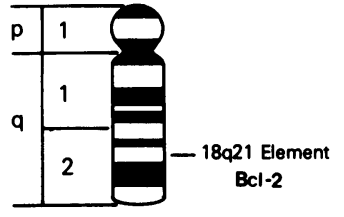

18

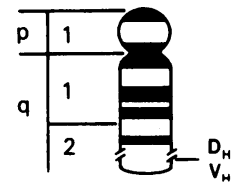

der 18
Figure 2. Typical follicular lymphoma with $\mathrm{t}(14 ; 18)$ (q32:q21) translocation. The normal chromosome 14 contains the $V / D / J$ rearrangement responsible for immunoglobulin production. The derivative (der) 14 chromosome has introduced a new B cell-associated gene from $18 \mathrm{q} 21(B c l-2)$ into $J_{H}$ near the enhancer $(E)$ element. The reciprocal (der) 18 partner has received $D_{H}$ and $V_{H}$ portions of chromosome 14.

highly conserved recombinatorial signals exist at this site on chromosome 18; instead, analysis of both reciprocal chromosomal partners favors a random double-stranded DNA breakage event upon 18 (52). Yet, such breakpoint clustering must have a functional role. Consequently, these breaks on 18 were shown to interrupt a $3^{\prime}$ exon of a B cell-associated gene (Bcl-2) generating a fusion transcript with the $\mathrm{Ig} \mathrm{J}_{\mathrm{H}}$ region (53-55). The normal Bcl-2 gene is highly transcribed at a pre-B cell stage of development when this translocation occurs. The RNA remains elevated in $t(14 ; 18)$ bearing mature $B$ cell neoplasms in contrast to its normal down-regulation in other mature B cells (55).

The clustering of the $t(14 ; 18)$ breakpoints creates unique rearrangements representing the site of chromosomal juncture. This event can be exploited to refine lymphoma cytogenetics and has revealed that many $14 \mathrm{q}^{+}$lymphomas lacking obvious reciprocal chromosomal partners are $t(14 ; 18)$ when examined for such DNA rearrangements (56). In addition, some of the $J_{H}$ rearrangements found in $\mathrm{T}$ cell neoplasms have proven to be

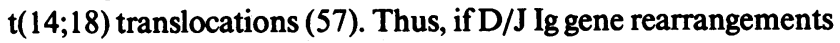
can spillover to the $\mathrm{T}$ cell lineage, it appears that $\mathrm{B}$ cell-associated translocations can also occur at this site within $T$ cells. However, the malignancy-associated translocations in $T$ cells classically occur at the TCR loci. The $\alpha$ TCR at $14 \mathrm{q} 11$ is particularly prone to translocation (58). Thus, an overwhelming association exists between TCR translocations in T cells and Ig gene translocations in B cells. The high incidence of these translocations in tumors, and of their absence from normal cellular counterparts, leaves no doubt about their major role in malignancy. However, questions do exist as to whether their high degree of lineage association is a primary or secondary event. Do these translocations occur early in development at the time of Ig or TCR assembly and in a prospective fashion direct the neoplasia into a particular cell type? Alternatively, do such translocations secondarily select these loci because they have already rearranged and are vulnerable to further recombination in cells already committed to the B or T lineage? Rare examples of inherited, familial transloca-
Table I. Uses of Gene Rearrangement in Hematopathology

1. Distinguish clonal from polyclonal lymphoproliferations.

2. Determine the B or $T$ cell identity of malignancies with admixed normal cells.

3. Determine the genetic lineage of neoplasms lacking definitive surface antigens.

4. Determine the developmental stage of early $B$ or $T$ cell precursors.

5. Identify unanticipated stages of development not appreciated within normal cell populations.

6. Tumor-specific markers to follow the natural history of neoplasms.

7. Search for minimal residual disease during clinical remission.

8. Molecularly mark chromosomal translocations and identify new transforming genes.

tions that involve these receptor loci do exist. These experiments of nature together with the introduction of isolated genes transgenically into the germline of mice should help to unravel the prospective ability of translocations to cause neoplasia and dictate its cellular type $(59,60)$.

\section{Future challenges}

The application of recombinant DNA technology to human lymphoid neoplasms has resolved many uncertain issues and made it possible to approach many more (Table I). Neoplastic cells are clonal expansions at distinct developmental steps and thus provide multiple copies of the somatic alterations that result in transformation. Major challenges remain in utilizing these events to better define tumor pathogenesis, refine diagnosis, predict prognosis, and improve therapy. Improved sensitivity in identifying minimal residual disease and better specificity in directing therapy to such neoplastic cells is sorely needed. The creation of new amino acids at the site of the Bcr-Abl fusion peptide in chronic myelogenous leukemia provides one example of a tumor specific molecule with which to develop new approaches (61). Large prospective studies are needed to determine whether any of the molecular refinements in pinpointing lineage and stage of development are of prognostic significance. The molecular definition of chromosomal breakpoints may allow routine cytogenetics to be essentially replaced with distinct molecular characterizations performed by Southern blot, pulsed field gel electrophoresis, and chromosomal in situ hybridization (62). Finally, the most important goal would be the capacity to precisely define all the transformation events within individual neoplasms. This information may hold the key to improving predictions of biologic behavior and will perhaps form the basis of tomorrow's therapies.

\section{References}

1. Foon, K. A., R. W. Schroff, and R. P. Gale. 1982. Blood. 60:119.

2. Leder, P. 1982. Sci. Am. (Lond.). 246:102-115.

3. Tonegawa, S. 1983. Nature (Lond.). 302:575-581.

4. Kronenberg, M., G. Siu, L. E. Hood, and N. Shastri. 1986. Annu. Rev. Immunol. 4:529-591.

5. Braylan, R. C., and H. Rappaport. 1973. Blood 42:579-589.

6. Levy, R., R. Warnke, R. F. Dorfman, and J. Haimovich. 1977. J. Exp. Med. 145:1014-1028.

7. Korsmeyer, S. J., P. A. Hieter, S. O. Sharrow, C. K. Goldman, P. Leder, and T. A. Waldmann. 1982. J. Exp. Med. 156:975-985. 
8. Arnold, A., J. Cossman, A. Bakhshi, E. S. Jaffe, T. A. Waldmann, and S. J. Korsmeyer. 1983. N. Engl. J. Med. 309:1593-1599.

9. Cleary, M. I., R. Warnke, and J. Sklar. 1984. N. Engl. J. Med. 310:477-482.

10. Hieter, P. A., G. F. Hollis, S. J. Korsmeyer, T. A. Waldmann, and P. Leder. 1981. Nature (Lond.). 294:536-540.

11. Minden, M. D., T. Toyonaga, K. Ha, Y. Yanagi, B. Chin, E. Gelfand, and T. W. Mak. 1985. Proc. Natl. Acad. Sci. USA. 82:12241227.

12. Waldmann, T. A., M. M. Davis, K. F., Bongiovanni, and S. J. Korsmeyer. 1985. N. Engl. J. Med. 313:776-783.

13. Flug, E., P. G. Pelicci, F. Bonetti, D. M. Knowles, and R. DallaFavera. 1985. Proc. Natl. Acad. Sci. USA. 82:3460-3464.

14. Aisenberg, A. C., T. G. Krontiris, T. W. Mak, and B. M. Wilkes. 1985. N. Engl. J. Med. 313:529-533.

15. Weiss, L. M., E. Hu, G. S. Wood, C. Moulds, M. L. Cleary, R. Warnke, and J. Sklar. 1985. N. Engl. J. Med. 313:539-544.

16. Bertness, V., I. Kirsch, G. Hollis, B. Johnson, and P. A. Bunn. 1985. N. Engl. J. Med. 313:534-538.

17. Korsmeyer, S. J., P. A. Hieter, J. V. Ravetch, D. G. Poplack, T. A. Waldmann, and P. Leder. 1981. Proc. Natl. Acad. Sci. USA. 78: 7096-7100.

18. Hieter, P. A., S. O. Sharrow, T. W. LeBien, J. H. Kersey, D. G. Poplack, P. Leder, and T. A. Waldmann. 1983. J. Clin. Invest. 71:301313.

19. Siminovitch, K. A., A. Bakhshi, P. Goldman, and S. J. Korsmeyer. 1985. Nature (Lond.). 316:260-262.

20. Raulet, D. H., R. D. Garman, H. Saito, and S. Tonegawa. 1985. Nature (Lond.). 314:103-107.

21. Snodgrass, H. R., P. Kisielow, M. Kiefer, M. Steinmetz, and H. von Boehmer. 1985. Nature (Lond.). 313:592-595.

22. Haars, R., M. Kronenberg, W. M. Gallatin, I. L. Weissman, F. L. Owen, and L. Hood. 1986. J. Exp. Med. 164:1-24.

23. Sangster, R. N., J. Minowada, N. Suciu-Foca, M. Minden, and T. W. Mak. 1986. J. Exp. Med. 164:1491-1508.

24. Wright, J. J., C. A. Felix, S. J. Korsmeyer, G. Reaman, D. Cole, P. Goldman, and D. G. Poplack. 1986. Proc. Am. Soc. Clin. Oncol. 5: 18. (Abstr.)

25. Brenner, M. B., J. McLean, D. P. Dialynas, J. L. Strominger, J. A. Smith, F. L. Owen, J. G. Seidman, S. Ip, F. Rosen, and M. S. Krangel. 1986. Nature (Lond.). 322:145-149.

26. Bank, I., R. A. DePinho, M. B. Brenner, J. Cassimeris, F. W. Alt, and L. Chess. 1986. Nature (Lond.). 322:179-181.

27. Sakano, H., Y. Kurosawa, M. Weigert, and S. Tonegawa. 1981. Nature (Lond.). 290:562-565.

28. Pelicci, P. G., D. M. Knowles, and R. Dalla-Favera. 1985. J. Exp. Med. 162:1015-1024.

29. Tawa, A., N. Hozumi, M. Minden, T. W. Mak, and E. W. Gelfand. 1985. N. Engl. J. Med. 313:1033-1037.

30. Yancopoulos, G. D., T. K. Blackwell, H. Suh, L. Hood, and F. W. Alt. 1986. Cell. 44:251-259.

31. Greaves, M. F., L. C. Chan, A. J. W. Furley, S. M. Watt, and H. V. Molgaard. 1986. Blood. 67:1-11.

32. Nadler, L. M., S. J. Korsmeyer, K. C. Anderson, A. W. Boyd, B. Slaughenhoupt, E. Park, J. Jensen, F. Coral, R. J. Mayer, S. E. Sallan, J. Ritz, and S. F. Schlossman. 1984. J. Clin. Invest. 74:332-340.

33. Bakhshi, A., J. Minowada, A. Arnold, J. Cossman, J. P. Jensen, J. Whang-Peng, T. A. Waldmann, and S. J. Korsmeyer. 1983. N. Engl. J. Med. 309:826-831.
34. Alt, F. W., and D. Baltimore. 1982. Proc. Natl. Acad. Sci. USA 79:4118-4122

35. Sklar, J., M. L. Cleary, K. Thielemans, J. Gralow, R. Warnke, and R. Levy. 1984. N. Engl. J. Med. 311:20-27.

36. Wright, J. J., D. G. Poplack, A. Bakhshi, G. Reaman, D. Cole, J. P. Jensen, and S. J. Korsmeyer. 1986. J. Clin. Oncol. In press.

37. Raffeld, M., J. J. Wright, N. Lipford, J. Cossman, A. Bakhshi, and S. J. Korsmeyer. 1986. Cancer Res. In press.

38. Siegelman, M. H., M. L. Cleary, R. Warnke, and J. Sklar. 1985. J. Exp. Med. 161:850-863.

39. Cleary, M. L., T. C. Meeker, S. Levy, E. Lee, M. Trela, J. Sklar, and R. Levy. 1986. Cell. 44:97-106.

40. Zehnbauer, B. A., D. M. Pardoll, P. J. Burke, M. L. Graham, and B. Vogelstein. 1986. Blood. 67:835-838.

41. Klein, G. 1981. Nature (Lond.). 294:313-318.

42. Kirsch, I. R., C. C. Morton, K. Nakahara, and P. Leder. 1982. Science (Wash. DC). 216:301-303.

43. Dalla-Favera, R., M. Bregni, J. Erikson, D. Patterson, R. C. Gallo, and C. M. Croce. 1982. Proc. Natl. Acad. Sci. USA. 79:7824-7827.

44. Adams, J. M., S. Gerondakis, and E. Webb. 1983. Proc. Natl. Acad. Sci. USA. 80:1982-1986.

45. Taub, R., I, Kirsch, C. Morton, G. Lenoir, D. Swan, S. Tronick, S. Aaronson, and P. Leder. 1982. Proc. Natl. Acad. Sci. USA. 79:78377841.

46. Lenoir, G. M., J. L. Preud'homme, A. Bernheim, and R. Berger. 1982. Nature (Lond.). 298:474-476.

47. Nishikura, K., A. Ar-Rushdi, J. Erikson, R. Watt, G. Rovera, and C. M. Croce. 1983. Proc. Natl. Acad. Sci. USA. 80:4822-4826.

48. Yunis, J. J. 1983. Science (Wash. DC). 221:227-236.

49. Tsujimoto, Y., L. R. Finger, J. J. Yunis, P. Nowell, and C. M. Croce. 1984. Science (Wash. DC). 226:1097-1099.

50. Bakhshi, A., J. P. Jensen, P. Goldman, J. J. Wright, O. W. McBride, A. L. Epstein, and S. J. Korsmeyer. 1985. Cell. 41:899-906. 51. Cleary, M. L., and J. Sklar. 1985. Proc. Natl. Acad. Sci. USA. 82:7439-7444.

52. Bakhshi, A., J. J. Wright, W. Graninger, M. Seto, J. Cossman, J. P. Jensen, P. Goldman, and S. J. Korsmeyer. 1986. Proc. Natl. Acad. Sci. USA. In press.

53. Cleary, M. L., S. D. Smith, and J. Sklar. 1986. Cell. 47:19-28.

54. Tsujimoto, Y., and C. M. Croce. 1986. Proc. Natl. Acad. Sci. USA. 83:5214-5218.

55. Graninger, W., P. Goldman, M. Seto, J. Wright, A. Bakhshi, and S. J. Korsmeyer. 1986. Fed. Proc. 45:986.

56. Lipford, E., J. J. Wright, W. Urba, J. Whang-Peng, I. R. Kirsch, M. Raffeld, J. Cossman, D. L. Longo, A. Bakhshi, and S. J. Korsmeyer. 1986. Clin. Res. 34:565A. (Abstr.)

57. Wright, J., A. Bakhshi, T. Han, F. Cabanillas, P. Goldman, and S. J. Korsmeyer. 1986. Clin. Res. 34:474A. (Abstr.)

58. Erikson, J., D. L. Williams, J. Finan, P. C. Nowell, and C. M. Croce. 1985. Science (Wash. DC). 229:784-6.

59. Leder, A., P. K. Pattengale, A. Kuo, T. A. Stewart, and P. Leder. 1986. Cell. 45:485-495.

60. Adams, J. M., A. W. Harris, C. A. Pinkert, L. M. Corcoran, W. S. Alexander, S. Cory, R. D. Palmiter, and R. L. Brinster. 1985. Nature (Lond.). 318:533-538.

61. Shtivelman, E., B. Lifshitz, R. P. Gale, and E. Canaani. 1985. Nature (Lond.). 315:550-554.

62. Carle, G. F., M. Frank, and M. V. Olson. 1986. Science (Wash. DC). 232:66-68 